\title{
Lactadherin blocks thrombosis and hemostasis in vivo: correlation with platelet phosphatidylserine exposure
}

\author{
J. SHI, * S. W. PIPE, $\dagger$ J. T. RASMUSSEN, $+C$. W. HEEGAARD $\$$ and G. E. GILBERT* \\ *Department of Medicine, VA Boston Healthcare System, Brigham and Women's Hospital and Harvard Medical School, Boston, MA; \\ $\dagger$ Department of Pediatrics, University of Michigan, Ann Arbor, MI, USA; and \$Protein Chemistry Laboratory, Department of Molecular \\ Biology, University of Aarhus, Aarhus, Denmark
}

To cite this article: Shi J, Pipe SW, Rasmussen JT, Heegaard CW, Gilbert GE. Lactadherin blocks thrombosis and hemostasis in vivo: correlation with platelet phosphatidylserine exposure. J Thromb Haemost 2008; 6: 1167-74.

Summary. Background: Platelet membrane phosphatidylserine (PS) is considered to be essential for hemostasis and thrombosis, but the in vivo topography of platelet PS has not been characterized. We hypothesized that platelet PS exposure would be identified on adherent platelets at the site of vascular injury and that blockade of PS would impede hemostasis and thrombosis. Objective: To localize and estimate the extent of platelet PS exposure and evaluate the impact of PS blockade in vivo. Methods: Lactadherin, a PS-binding milk protein, was utilized together with annexin $\mathrm{V}$ to detect both partial and complete membrane PS exposure on platelets in a mouse model of thrombosis and to evaluate the functional need for PS. Preliminary experiments were performed with synthetic membranes and with purified platelets. Results: The number of lactadherin-binding sites on synthetic membranes was proportional to PS content, whereas annexin V required a threshold of $2.5-8 \%$ PS. Approximately $95 \%$ of thrombin-stimulated platelets exposed PS, but the quantity was below the threshold for annexin $\mathrm{V}$ binding at physiologic $\mathrm{Ca}^{2+}$ concentrations. In mice, most adherent and aggregated platelets on the walls of ferric chloride-treated mesenteric veins exposed low levels of PS, rather than having complete exposure. In mice, blockade of PS with lactadherin inhibited platelet prothrombinase and factor Xase activity, and prolonged tail bleeding time and the time to carotid artery thrombosis. Conclusions: In vivo PS exposure contributes to both hemostasis and thrombosis. In this model of vascular injury, most platelets exhibit partial rather than complete PS exposure.

Keywords: annexin, factor V, factor VIII, lactadherin, phosphatidylserine, platelets.

Correspondence: Gary E Gilbert, VA Boston Healthcare Center, 1400 VFW Parkway, West Roxbury, MA 02132, USA.

Tel.: + 1857203 5252; fax: + 18572035592.

E-mail: ggilbert@rics.bwh.harvard.edu

Received 14 February 2008, accepted 25 April 2008

\section{Introduction}

Current models of hemostasis and thrombosis indicate that phospholipid, and particularly phosphatidylserine (PS), is an essential cofactor. Under quiescent conditions, PS is largely hidden from circulating blood on the inner leaflet of the plasma membranes of platelets. However, platelets undergo a terminal, apoptosis-like process and redistribute PS to the outer leaflet of the plasma membrane [1-3]. In addition, viable platelets can expose limited quantities of PS to support coagulation reactions [4,5]. However, the extent and location of platelet PS exposure in vivo and the necessity of this for hemostasis and thrombosis has been largely inferred from in vitro work, rather than empirically tested.

PS exposure is frequently detected through binding of fluorescently labeled annexin V [6]. The extent to which annexin $\mathrm{V}$ binds to a membrane is a complex function related to the free annexin V concentration, membrane PS content, phosphatidylethanolamine (PE) content, and ambient $\mathrm{Ca}^{2+}$ concentration (see Supplementary material). The established studies do not include an explicit correlation of annexin $\mathrm{V}$ binding with cell membrane PS content. Thus, absence of annexin $\mathrm{V}$ binding is sometimes interpreted as absence of PS, whereas some in vitro studies suggest the possibility that PS might be exposed on stimulated platelets $[7,8]$ but remain below the threshold value required for annexin $\mathrm{V}$ binding.

Lactadherin is also a PS-binding protein and has a domain structure that includes two epithelial growth factor-like domains, and two lectin-type ' $\mathrm{C}$ ' domains that are homologous with the PS-binding domains of blood clotting factor VIII [9]. The biology of lactadherin has been emerging from recent in vivo studies (see Supplementary material). Lactadherin exhibits stereoselective interaction with phospho-L-serine of PS [10,11], and PS binding is predominantly mediated by the $\mathrm{C} 2$ domain [10]. In contrast to that of annexin $\mathrm{V}$, membrane binding of lactadherin appears to be proportional to PS content, is independent of $\mathrm{Ca}^{2+}$ concentration, and is independent of membrane PE content [12]. We have recently reported that fluorescently labeled lactadherin can be utilized to provide sensitive information about the extent and location of 
PS exposure on cells early in apoptosis [13]. We have also utilized the $\mathrm{C} 2$ domain of lactadherin to localize intracellular PS in quiescent and stimulated cells [14].

This work aimed to investigate the hypothesis that platelet PS exposure accompanies vascular injury and is required for hemostasis and thrombosis. The results indicate that lactadherin and annexin $\mathrm{V}$ can be utilized to detect and estimate the extent of PS exposure. In vivo studies confirmed the presence of primarily partial platelet PS exposure on adherent platelets in the course of venous thrombus formation and the necessity of exposed PS for hemostasis and thrombosis.

\section{Materials and methods}

See Supplementary material.

\section{Results}

We hypothesized that the contrasting PS-binding properties of lactadherin and annexin $\mathrm{V}$ would enable in vivo detection of exposed platelet PS as well as an estimate of the extent to which PS is exposed [13]. Preliminary studies were performed with synthetic membranes supported by $2-\mu \mathrm{m}$ glass microspheres [15] (Fig. 1). The $\mathrm{Ca}^{2+}$ concentration was $1.5 \mathrm{~mm}$, near the upper limit of $\mathrm{Ca}^{2+}$ in blood plasma [16]. First, we assumed that PE exposure would not increase with PS exposure. Thus, the PS content was varied from $0 \%$ to $12 \%$ in membranes with the PE content fixed at 2\% (Fig. 1A). Bound lactadherin increased with PS content and approached a plateau at $8 \%$ PS. In contrast, annexin $\mathrm{V}$ binding was evident only when the PS content was at least $8 \%$ (Fig. 1B). When the ambient lactadherin concentration was $4 \mathrm{nM}$, the quantity bound to membranes was proportional to PS content for PS $<4 \%$ but tapered towards a plateau at higher concentrations (Fig. 1C).

Assuming that PE might be exposed more extensively than PS, we synthesized membranes with a PE/PS ratio of $4: 1$ (Fig. 1D). Binding curves indicated that the number of lactadherin-binding sites remained proportional to PS content for PS $<8 \%$, as previously reported [13]. When the ambient concentration of lactadherin or annexin $V$ was $4 \mathrm{~nm}$, the presence of excess PE reduced the PS threshold for annexin V to $\sim 2.5 \%$ PS vs. $8 \%$ PS (Fig. 1C). These results predict that lactadherin will bind to cell membranes with PS content $\geq 0.5 \%$ and be largely unaffected by PE content. Annexin V is predicted to bind when the PS content exceeds $2.5-8 \%$, depending on the PE content. Annexin V supplied by three vendors, conjugated with three different fluorophores, gave equivalent results. A conjugate of annexin V-Alexa Fluor 647 prepared in our laboratory was utilized for the experiments displayed in Figs 2 and 3.

\section{Partial and complete platelet PS exposure}

Experiments with purified platelets were performed to characterize PS exposure by incubation with lactadherin and annexin V. Stimulation of platelets with a calcium ionophore led to two
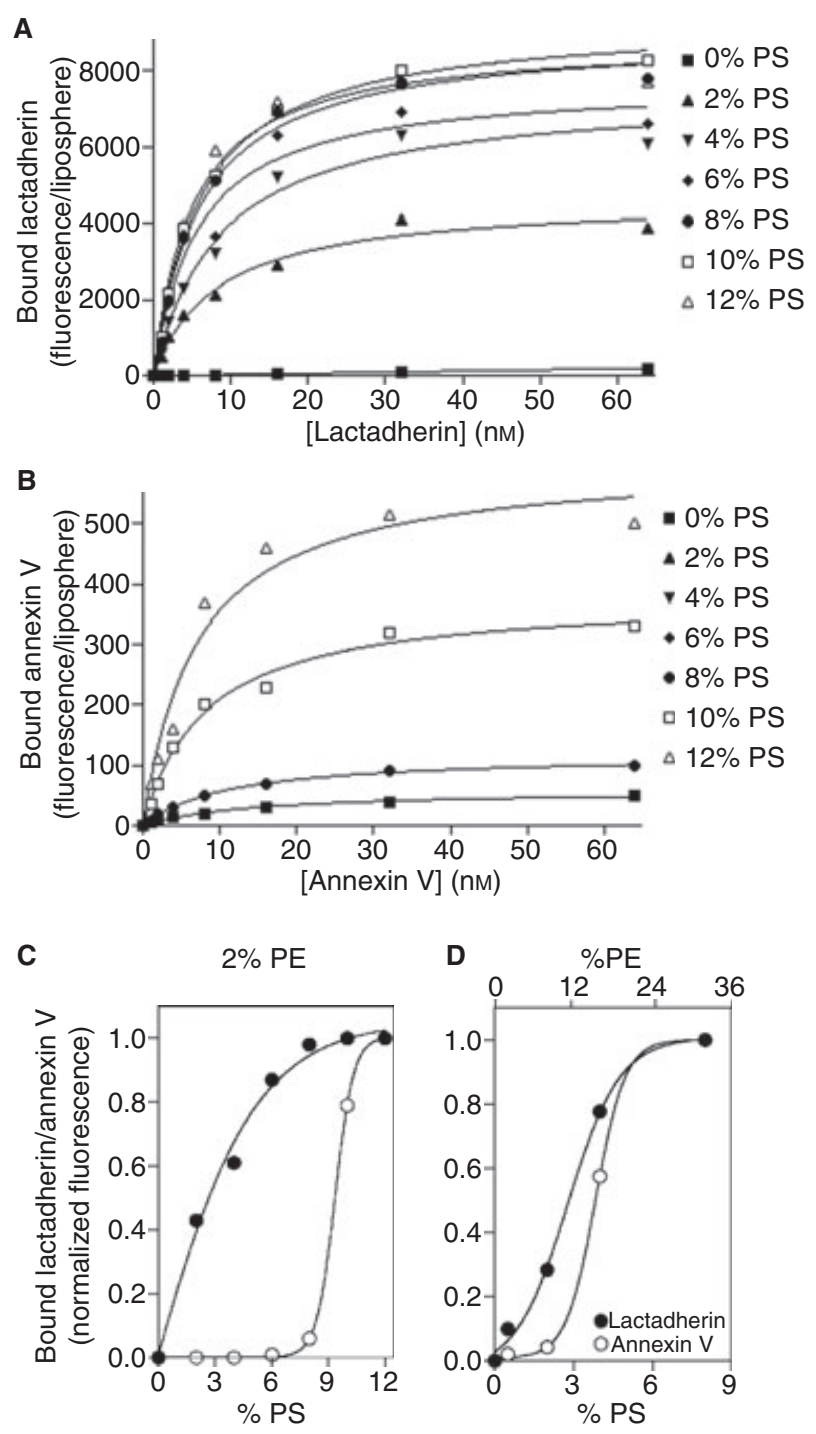

Fig. 1. Relationship of membrane phosphatidylserine (PS) content to lactadherin and annexin V binding. Fluorescein-labeled lactadherin (A) or fluorescein-labeled annexin V (B) was mixed with lipospheres. Membranes had 0--12\% PS, 2\% phosphatidylethanolamine (PE) and the balance as phosphatidylcholine. Bound lactadherin or annexin $\mathrm{V}$ was evaluated by flow cytometry after $10 \mathrm{~min}$. Values for annexin $\mathrm{V}$ binding to membranes of $2 \%, 4 \%$ and $6 \%$ PS overlapped with $0 \%$ PS values, so that most data points for these curves are hidden by the filled squares for the $0 \%$ PS curve. (C) Four nanomolar fluorescein-labeled lactadherin $(\bullet)$ or annexin $\mathrm{V}(\mathrm{O})$ was incubated with lipospheres of various membrane compositions [from (A) and (B)]. Results are normalized to the quantity of bound annexin $\mathrm{V}$ or lactadherin at $12 \%$ PS. (D) The effect of increasing $\mathrm{PE}$ on binding was evaluated. Four nanomolar fluorescein-labeled lactadherin or annexin $\mathrm{V}$ was incubated with lipospheres of varying PS content with a PE/PS ratio of $4: 1$, the balance being PC.

levels of PS exposure (Fig. 2). Shear stress and mixing were minimized so that microparticles usually represented fewer than $10 \%$ of events $[17,18]$, and the detection gate was set to exclude microparticles, as previously described [7]. When the concentration of A23187 was $0.25 \mu \mathrm{M}$, most platelets bound lactadherin but not annexin V, suggesting that PS was exposed but that the PS content remained below the annexin V 

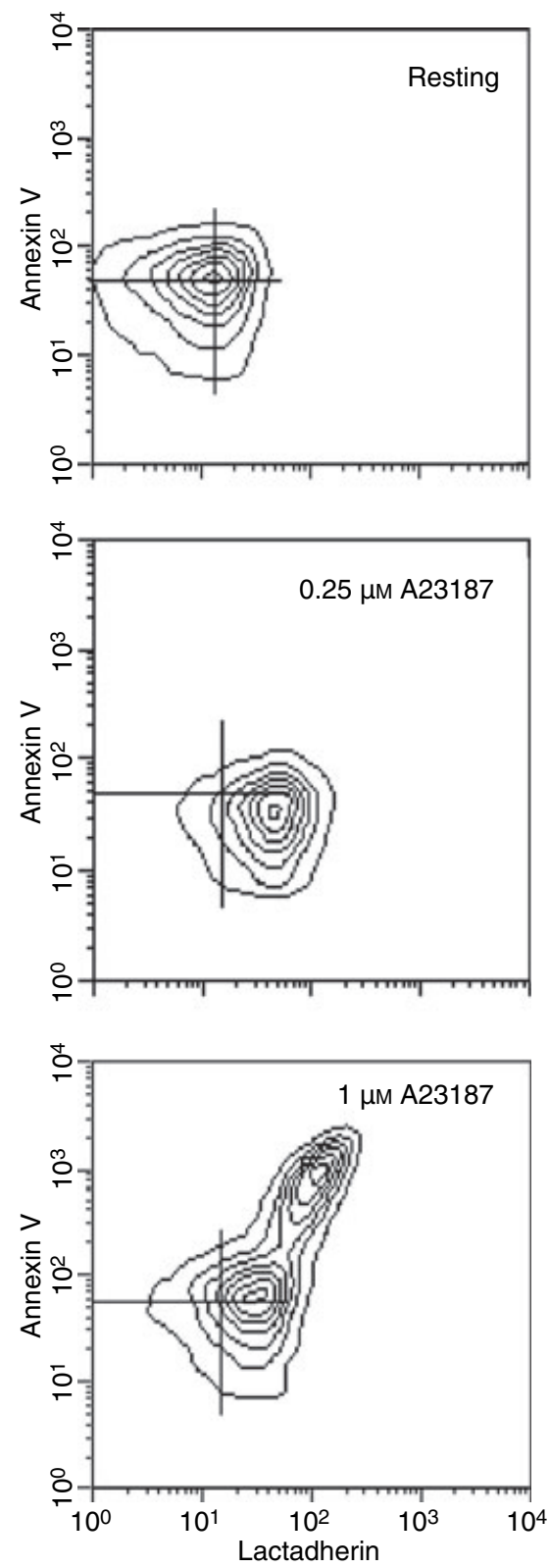

Fig. 2. Phosphatidylserine exposure on platelets stimulated with A23187. Fluorescein-labeled lactadherin, $4 \mathrm{~nm}$, and Alexa Fluor 647-annexin V, $4 \mathrm{~nm}$, were added to platelets, $1 \times 10^{6} \mathrm{~mL}^{-1}$, and measurements of platelet fluorescence were obtained after addition of buffer control (Resting) $0.25 \mu \mathrm{M}$, or $1.0 \mu \mathrm{M}$ A23187. Five minutes after exposure to A23187, platelets exhibited increased lactadherin binding, and at $1.0 \mu \mathrm{M}$ A23187, increased annexin $\mathrm{V}$ binding and a further increase in lactadherin binding. Contour lines indicate $15 \%$ linear increments of event density. Cross-hairs mark modal fluorescence for resting platelets.

threshold. The width and shape of the platelet contour plot did not broaden, indicating that most of the platelet population exposed PS to a similar degree. A few platelets stained brightly with both lactadherin and annexin V, as shown in Fig. 3. This minor population was excluded from the linear contour plots of the upper and middle panels by the data reduction algorithm (threshold of $1 \%$ event density). Staining with either annexin $\mathrm{V}$ or lactadherin, in the absence of the other, yielded equivalent results, indicating that competition between the two proteins did not explain the lack of binding by annexin V. In addition, control experiments showed that these concentrations of lactadherin and annexin $\mathrm{V}$ do not influence the binding of the other by more than $20 \%$ on synthetic membranes [13].

When the concentration of A23187 was increased to $1 \mu \mathrm{M}$, approximately half of the platelets bound much more lactadherin and annexin V. At higher concentrations of A23187, all of the platelets bound both lactadherin and annexin V (not shown). Control experiments indicated that binding of lactadherin is mediated via the PS-binding $\mathrm{C} 2$ domain rather than the integrin-binding epidermal growth factor domain for both levels of PS exposure (supplementary Fig. S1). Plateletsupported factor Xase activity and prothrombinase activity were inhibited $>95 \%$ by lactadherin (supplementary Fig. S2). Inhibition by annexin $\mathrm{V}$ was similar to that by lactadherin at concentrations up to $20 \mathrm{~nm}$ for platelets stimulated with the highest concentrations of ionophore, but annexin $\mathrm{V}$ was otherwise less effective than lactadherin. Thus, these data confirm that platelets have capacity for regulated, partial PS exposure as well as complete PS exposure. Furthermore, the data confirm that exposed platelet PS is necessary to support procoagulant activity at both levels of PS exposure.

\section{Partial PS exposure on platelets stimulated by thrombin}

We investigated whether lactadherin binding would identify limited PS exposure on platelets stimulated by thrombin, analogously to low concentrations of A23187 (Fig. 3A). Thrombin caused exposure of binding sites for lactadherin, although the intensity of staining was less than that produced by the lower concentrations of ionophore (Fig. 3A). Binding of annexin $\mathrm{V}$ to the major platelet population was not detected. Subsequent experiments, performed with the protease-activated receptor-1 (PAR-1) agonist, thrombin receptor activation peptide (TRAP), gave equivalent lactadherin binding (Fig. 3B). Control experiments, analogous to those shown in supplementary Fig. S1, confirmed that lactadherin binds to TRAPstimulated platelets via the PS-binding $\mathrm{C} 2$ domain (not shown). Thus, PAR-1 stimulation is sufficient to cause PS exposure and was utilized as an agonist for the experiments displayed in Fig. 5.

A small fraction of platelets were strongly positive for both annexin V and lactadherin (region M2, Fig. 3A). This fraction ranged from $0.5 \%$ to $1.5 \%$ for unstimulated platelets and from $0.8 \%$ to $3 \%$ for platelets stimulated with TRAP, thrombin, or $0.1-0.5 \mu \mathrm{M}$ A23187. The fraction of platelets with complete PS exposure was only minimally influenced by purification on a density gradient vs. simple dilution of platelet-rich plasma (not shown). Thus, the platelets with maximal PS exposure did not appear to result from the purification procedure.

\section{In vivo pattern of platelet PS exposure is predominantly partial}

We performed experiments to determine the location and extent of PS exposure on platelets during in vivo thrombosis. 
A

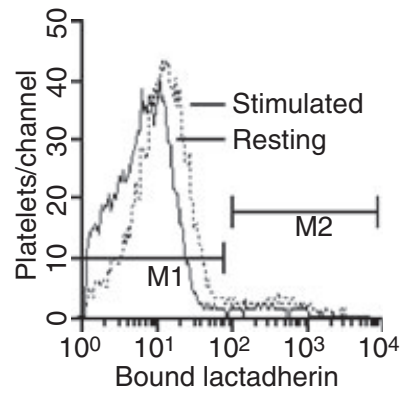

(Fluorescence)

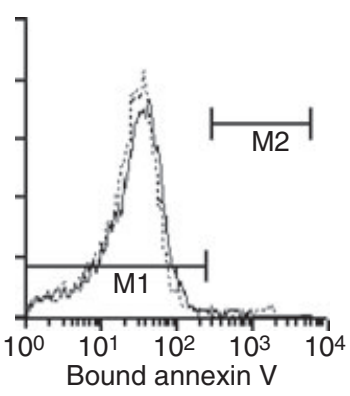

(Fluorescence)
B

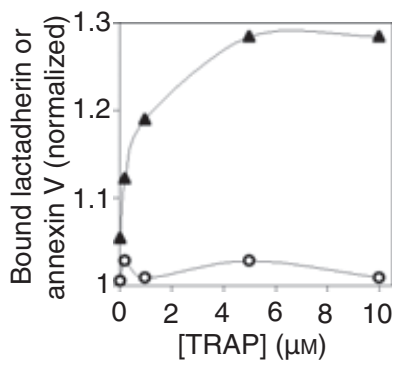

Fig. 3. Expression of phosphatidylserine (PS) on platelets stimulated via the protease-activated receptor-1 thrombin receptor. (A) Fluorescein-labeled lactadherin and Alexa Fluor 647-labeled annexin V were mixed with the platelets prior to the addition of 10 nM thrombin, as in Fig 2. Gates M1 and M2 indicate platelets that were classified as having regulated PS exposure vs. complete exposure. (B) Various quantities of thrombin receptor activation peptide (TRAP) were added to platelets to evaluate the optimal concentration for stimulating exposure of PS detected with lactadherin $(\mathbf{\Delta})$ and annexin $\mathrm{V}(\mathrm{O})$.

Anesthetized mice were injected with $1 \mu \mathrm{g}$ each of lactadherin and annexin $\mathrm{V}$ immediately prior to induction of mesenteric vein thrombosis with ferric chloride (Fig. 4). The concentrations of both proteins were sufficiently low that the rates of thrombosis and hemostasis were unaffected (see below and Fig. 5). At this time point, most veins remained patent (Fig. 4A), but intramural hemorrhage and early luminal thrombosis were underway (Fig. 4B).

Fibrin(ogen) was identified along the injured vessel wall and within platelet aggregates (Fig. 4C, red color). Platelets were located along the injured vessel wall as well as on fibrin strands within the lumen (Fig. 4D). Lactadherin colocalized with platelets and the elevated endothelium along the vessel wall (Fig. 4E). However, the platelet aggregates in the vessel lumen (Fig. 4F) and platelets along fibrin strands did not detectably stain. Unlike lactadherin, annexin V did not detectably stain either platelets or other components of the developing thrombi. Serial dilutions of lactadherin vs. annexin $\mathrm{V}$ in a dot blot calibration assay indicated that the polyclonal antibody for annexin V was within 4-fold of the sensitivity for lactadherin. These results indicate that platelets adjacent to an injured vessel expose more accessible PS than those that adhere to other platelets or to fibrin in the lumen. Platelets from control mice that did not receive lactadherin and annexin $\mathrm{V}$ injections did not stain with these antibodies, indicating a lack of crossreaction with murine proteins.

Use of the TrueBlue horseradish peroxidase substrate, as previously described for in vivo detection of annexin $\mathrm{V}$ binding [19], enhanced the sensitivity of the immunohistochemistry. With this dye, annexin $\mathrm{V}$ was detected on occasional platelets and on some endothelial cells (Fig. 4G,H). However, the majority of adherent and aggregated platelets did not stain for annexin V. Using the TrueBlue substrate, lactadherin was detected on virtually all adherent and aggregated platelets (Fig. 4I,J). Platelet aggregates were costained with anti-CD41 to identify platelets in complex thrombi (Fig. 4I). Lactadherin staining was also observed on occasional endothelial cells and a small number of trapped red cells (Fig. 4J). The number of adherent and aggregated platelets varied considerably in different vessels, as did the quantity of fibrin. However, the pattern of platelet and endothelial staining by lactadherin and annexin $\mathrm{V}$ was consistent throughout the sections. Taken together, these immunohistochemical data indicate that most adherent and aggregated platelets expose PS below the threshold for annexin $\mathrm{V}$ binding and that platelets close to the vessel wall expose more PS than those that are more than approximately five platelet diameters from the wall.

\section{Inhibition of thrombosis and hemostasis by PS blockade}

The capacity of lactadherin and annexin $\mathrm{V}$ to inhibit the procoagulant activity of stimulated platelets was investigated (Fig. 5). Platelets were stimulated with TRAP, and the inhibitory effects on FXa production and thrombin production were evaluated. Lactadherin inhibited more than $99 \%$ of FXa and more than $98 \%$ of thrombin production. In contrast, annexin V inhibition approached a plateau at somewhat over $90 \%$ inhibition. Thus, accessible platelet membrane PS is necessary for efficient procoagulant activity in vitro.

Prior results from our laboratory indicated that plateletdependent clotting of whole blood is inhibited by lactadherin [12]. Thus, we hypothesized that lactadherin would exhibit in vivo anticoagulant activity. To test this hypothesis, experiments were performed on anesthetized mice (Fig. 5C,D). Mice were injected with $0.21 \mathrm{nmol}$ of lactadherin or annexin $\mathrm{V}$ prior to transection of the tail. Blood loss was approximately 4-fold higher following lactadherin injection. The results were equivalent when analyzed according to the bleeding times. Equimolar doses of annexin $\mathrm{V}$ did not prolong the tail bleeding time. Thus, access of blood to membrane PS is required for normal hemostasis.

To evaluate the necessity of PS exposure for in vivo thrombosis, lactadherin was evaluated in a carotid artery occlusion model (Fig. 5D). Lactadherin doses $\leq 0.12 \mathrm{nmol}$ did not affect time to occlusion. In contrast, doses of $0.16-$ $0.42 \mathrm{nmol}$ prolonged time to occlusion from approximately 37 min, consistent with prior reports [20], to approximately $80 \mathrm{~min}$. Four of the treated mice had not yet occluded their carotid arteries when the experiment was terminated after 90 min (arrows). Carotid artery occlusion experiments were 

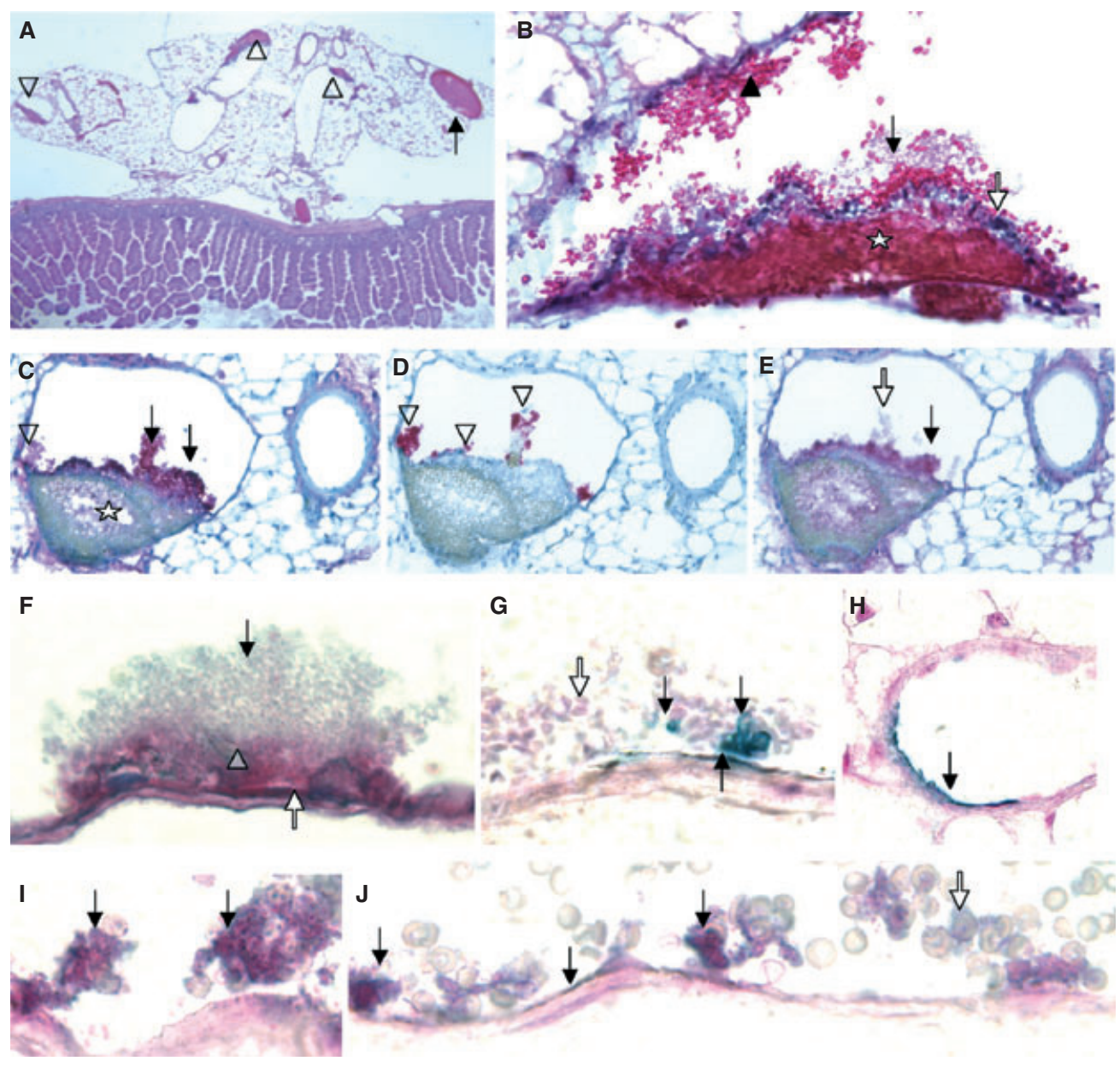

Fig. 4. Phosphatidylserine exposure in mouse mesenteric venous thrombosis. (A) Hematoxylin and eosin-stained sections with intramural hemorrhage (open triangles) and congestion (arrow). At higher magnification (B), the intramural hemorrhage (open star) was distinguishable from the elevated endothelium (open arrow) aggregated platelets (closed arrow) and erythrocytes (closed triangle). Serial sections were stained with anti-fibrinogen/fibrin (C), anti-platelet (D) and anti-lactadherin antibodies (E) developed with the alkaline phosphatase Vector Red substrate. A layer of fibrinogen/fibrin (C, closed arrows) overlaid a mural hemorrhage (open star). Platelets (D) were scattered along the luminal surface of the thrombus (open triangles) as well as upon fibrinogen/fibrin strands extending into the lumen. Lactadherin staining (E) was strongest along the raised endothelium surface (closed arrow), including adherent platelets close to the wall. Platelets on fibrin strands did not stain detectably (open arrow) (E). Higher magnification of a large platelet aggregate (F) revealed staining of the endothelium (open arrow) and the aggregated platelets within 5--10 platelet diameters of the endothelium (open triangle). Platelets further from the wall did not stain detectably (closed arrow). Annexin V was detected with TrueBlue stain (G, H) on scattered platelets and endothelial cells (closed arrows). Most aggregated platelets did not stain detectably for annexin V (open arrow). Lactadherin staining with TrueBlue indicated that all of the aggregated platelets stained at a level that had been undetectable with Vector Red (I, J). Large platelet aggregates were marked by reticular lactadherin staining (I, closed arrows). The intense red of the platelet aggregates reflects costaining with anti-CD41 and Vector Red. A longitudinal vein section (J) illustrates staining of platelets and endothelium (closed arrows) and peripheral staining around scattered erythrocytes (open arrow). Displayed panels are representative of 30--40 thrombi examined in eight mice, treated in three groups.

also performed following injection of annexin V (data not shown). The degree of inhibition was substantially less than with lactadherin. However, the absence of comparative pharmacokinetic data for lactadherin vs. annexin V makes these data difficult to compare. The results confirm that blockade of PS exposure by lactadherin inhibits thrombosis as well as hemostasis.

\section{Discussion}

This article demonstrates that platelet PS exposure can be detected in vitro and in vivo with lactadherin and annexin $\mathrm{V}$.
Lactadherin binds to platelets with partial PS exposure, whereas both reagents bind to platelets that have complete PS exposure. Most adherent platelets in a murine thrombosis model stained with lactadherin but not annexin $\mathrm{V}$, indicating that PS exposure on most platelets was limited rather than complete. Prolongation of the tail bleeding time and the carotid artery occlusion time by lactadherin confirmed that in vivo access of blood coagulation proteins to PS-containing membrane contributes to both hemostasis and thrombosis.

FVIII and FV bind to PS in a stereoselective manner [21,22]. Binding of the activated proteins, together with the respective serine proteinases, FIXa and FXa, leads to condensation on 



Fig. 5. Inhibition of coagulation by phosphatidylserine-blocking proteins. Platelets were stimulated with $10 \mu \mathrm{M}$ thrombin receptor activation peptide for $10 \mathrm{~min}$, and the generation of factor Xa (A) or thrombin (B) was measured in the presence of various concentrations of lactadherin $(\mathbf{O})$ or annexin $\mathrm{V}(\Delta)$. Other experimental conditions were as described in the legend to supplementary Fig. S2. (C) The effect of lactadherin $(0.21 \mathrm{nmol})$ and annexin $\mathrm{V}(0.21 \mathrm{nmol})$ on the tail bleeding volume of mice was evaluated. Blood loss increased nearly 4-fold following lactadherin injection ( $P=0.001$, Mann-Whitney analysis) but was not significantly changed by annexin. (D) The effect of lactadherin on thrombosis was evaluated in a mouse carotid artery injury model. The time to occlusion was monitored. Four mice, treated with lactadherin, had not occluded carotid arteries at the termination of the 90-min experiment (arrows). Difference between means $P=0.03$ (Mann-Whitney analysis, assuming 90-min occlusion times for four non-occluding animals).

the membrane as well as allosteric activation of the assembled procoagulant complexes $[23,24]$. The data in this article indicate that blockade of platelet PS with lactadherin inhibits $>98 \%$ of activity for both the factor Xase and the prothrombinase complexes. Thus, competition by lactadherin for PS-binding sites probably contributes to the in vivo anticoagulant activity of lactadherin. We have recently solved the crystal structure of the lactadherin C2 domain and identified membraneinteractive amino acids [25]. Ongoing studies will seek to identify the interactions that are essential for the anticoagulant functionality.

Our data obtained with annexin $\mathrm{V}$ and lactadherin are consistent with prior reports. In studies of ex vivo and in vivo thrombus formation, platelets staining with annexin $\mathrm{V}$ were scattered near the periphery of platelet-fibrin aggregates, and fibrin generation in venules proceeded with no annexin $\mathrm{V}$ staining [26]. A preliminary report using different methodology indicated that annexin $\mathrm{V}$ does not localize to aggregated platelets at a site of vascular injury but stains the local endothelial cells [27]. The requirement of annexin $\mathrm{V}$ for membrane PS content $>1 \%$ was previously observed [28]. The absence of binding sites for annexin V on most thrombinstimulated platelets has also been reported [29]. Prior inhibition of platelet factor Xase activity and platelet prothrombinase was consistent with the degree of inhibition that we observed, although the experimental details were different [30-32]. Likewise, the binding of annexin $\mathrm{V}$ to platelets stimulated with $>1 \mu \mathrm{M}$ A23187 is consistent with prior reports, and confirmed that the annexin V that we utilized was intact [29]. A recent report also indicated that lactadherin binds purified, thrombin-stimulated platelets when little or no annexin V binding is demonstrable [33].

The in vivo anticoagulant activities of lactadherin and annexin $\mathrm{V}$ can be rationalized with the in vitro activities and with prior reports. Our data indicate that lactadherin inhibits hemostasis but annexin $\mathrm{V}$ has no impact at the doses tested. This is consistent with prior reports in which annexin $\mathrm{V}$ did not inhibit hemostasis in rats [34] or in rabbits [35,36]. These findings suggest that the greatest source of procoagulant activity for hemostasis comes from platelets with limited PS exposure. Our data indicate that annexin $\mathrm{V}$ inhibits carotid artery occlusion, although to a lesser degree than lactadherin. Prior data indicate that annexin $\mathrm{V}$ inhibits fibrin accumulation in mesenteric [34] and carotid arteries [36] as well as jugular veins [35]. Thus, annexin V appears to be a more effective anticoagulant in vascular injury models in which the endothelium and the adjacent layer of adherent platelets are likely to have complete PS exposure. Our in vitro data predict that lactadherin will be a more effective inhibitor of thrombotic occlusion than annexin $\mathrm{V}$ because it is a more effective inhibitor of FXa and thrombin generation. However, the best experimental comparison will require stable, comparable plasma concentrations of the two phospholipid-binding proteins.

Prior evidence has implied that thrombin-stimulated, intact platelets have the capacity to express PS in a limited, regulated fashion $[8,37,38]$ as well as the capacity to progress to complete, apoptosis-like PS exposure [1,3]. The extent of PS exposure elicited by calcium ionophore is equivalent to the extent of PS exposure when platelets are stimulated by both thrombin and collagen, underscoring the fact that maximal PS exposure occurs with both pathways of stimulation [1]. In addition, stimulated platelets can shed plasma membrane vesicles with complete PS exposure [39-41]. This study emphasized detection of PS exposure on platelets rather than on microparticles. For in vitro studies, microparticle formation was minimized by application of low sheer stress [18], and microparticles were excluded from flow cytometry analysis by selective gating. However, we looked for platelet microparticles in histologic sections as punctate regions staining for lactadherin and annexin V. These were not clearly identified. However, it remains possible that microparticles were present but that the number was too small to make them evident. Our in vitro data confirmed that $1.5-3 \%$ of platelets progressed to complete PS exposure in response to PAR-1 stimulation. Our in vivo data demonstrated a similar fraction of platelets that bound annexin $\mathrm{V}$ at the site of provoked venous thrombosis. The relative procoagulant contribution of the platelets with complete PS exposure is higher in vitro [42]. The relative procoagulant contribution in vivo will probably be clarified through additional studies with genetically altered animals [43] and, 
possibly, as future reagents are developed that selectively block PS-dependent coagulation reactions on PS-rich vs. PS-poor membranes.

Although lactadherin and annexin V bind to PS-containing membranes with similar affinities, each protein does not compete efficiently for the other's binding sites on synthetic membranes [13]. The similarity between the binding mechanism of lactadherin and that of blood coagulation proteins FV and FVIII correlates with the capacity to predictably inhibit coagulation reactions based on phospholipid membranes while reactions supported by some membranes are not inhibited effectively by annexin $\mathrm{V}[12]$. The data in this report suggest that platelet membranes may display procoagulant binding sites expressing low quantities of PS and/or with high degrees of curvature that support coagulation and are blocked by lactadherin but not annexin V. We note an apparent conflict between the absence of annexin $\mathrm{V}$ binding to thrombinstimulated platelets and the capacity of annexin $\mathrm{V}$ to inhibit $70-95 \%$ of platelet-dependent prothrombinase and factor Xase activity. Both results have been previously reported [29,32], so we do not believe that the apparent conflict is an artefact related to our experimental system. It is possible that the annexin $\mathrm{V}$ multimers that form at higher concentrations in the presence of $\mathrm{Ca}^{2+}$ bind to thrombin-stimulated platelets whereas annexin $\mathrm{V}$ monomers do not. It is also possible that annexin $\mathrm{V}$ has a low affinity for both platelet membranes and one or more proteins of the prothrombinase and factor Xase complexes. Thus, annexin V would bind and inhibit function only when the proteins of the enzyme complexes were present. It also remains possible that the in vivo staining and anticoagulant effects of both annexin $\mathrm{V}$ and lactadherin are further modified by plasma proteins. The current data are sufficient to conclude that lactadherin has the capacity to inhibit $>98 \%$ of prothrombinase and factor Xase activity related to platelets and that the inhibitory capacity of annexin $\mathrm{V}$ is lower.

In summary, lactadherin and annexin $\mathrm{V}$ can be used to estimate the extent of platelet PS exposure in vitro and in vivo. With this approach, we have found that most platelets at the site of vascular injury expose PS but that the extent of PS exposure remains below the threshold for annexin V binding. Thus, the platelet mechanism for regulating PS exposure following adhesion to sites of vascular injury could be a regulatory element of hemostasis and thrombosis. The capacity of lactadherin to efficiently block procoagulant PS enabled studies that confirmed that exposed PS is essential for normal hemostasis and thrombosis.

\section{Acknowledgements}

We are grateful to P. Price, J. Zhao and H. Miao for excellent technical assistance. A Merit Award from the Veterans Administration Healthcare System supported this work.

\section{Disclosure of Conflict of Interests}

The authors state that they have no conflict of interest.

\section{Supplementary material}

The following supplementary material can be found at http:// www.blackwell-synergy.com/loi/jth :

Experimental procedures.

Fig. S1. Lactadherin binding to platelets mediated by phosphatidylserine-binding $\mathrm{C} 2$ domain.

Fig. S2. Inhibition of platelet FXa and prothrombin production by lactadherin or annexin $\mathrm{V}$.

This material is available as part of the online article from http://www.blackwell-synergy.com/doi/abs/10.1111/j.15387836.2008.03010.x (This link will take you to the article abstract).

Please note: Blackwell Publishing are not responsible for the content or functionality of any supplementary materials supplied by the authors. Any queries (other than missing material) should be directed to the corresponding author for the article.

\section{References}

1 Alberio L, Safa O, Clemetson KJ, Esmon CT, Dale GL. Surface expression and functional characterization of alpha-granule factor $\mathrm{V}$ in human platelets: effects of ionophore A23187, thrombin, collagen, and convulxin. Blood 2000; 95: 1694-702.

2 Cohen Z, Wilson J, Ritter L, McDonagh P. Caspase inhibition decreases both platelet phosphatidylserine exposure and aggregation: caspase inhibition of platelets. Thromb Res 2004; 113: 387-93.

3 London FS, Marcinkiewicz M, Walsh PN. A subpopulation of platelets responds to thrombin- or SFLLRN-stimulation with binding sites for factor IXa. $J$ Biol Chem 2004; 279: 19854-9.

4 Bevers E, Comfurius P, Van Rijn J, Hemker H, Zwaal R. Generation of prothrombin-converting activity and the exposure of phosphatidylserine at the outer surface of platelets. Eur J Biochem 1982; 122: 429-36.

5 Williamson P, Bevers EM, Smeets EF, Comfurius P, Schlegel RA, Zwaal RFA. Continuous analysis of the mechanism of activated transbilayer lipid movement in platelets. Biochemistry 1995; 34: 1044855.

6 Koopman G, Reutelingsperger CP, Kuijten GA, Keehnen RM, Pals ST, van Oers MH. Annexin V for flow cytometric detection of phosphatidylserine expression on B cells undergoing apoptosis. Blood 1994; 84: 1415-20.

7 Phillips JE, Lord ST, Gilbert GE. Fibrin stimulates platelets to increase factor VIIIa binding site expression. J Thromb Haemost 2004; 2: 1806 15.

8 Ahmad SS, Rawala-Sheikh R, Ashby B, Walsh PN. Platelet receptormediated factor X activation by factor IXa: high-affinity factor IXa receptors induced by factor VIII are deficient on platelets in Scott syndrome. J Clin Invest 1989; 84: 824-8.

9 Stubbs J, Lekutis C, Singer K, Bui A, Yuzuki D, Srinivasan U, Parry G. cDNA cloning of a mouse mammary epithelial cell surface protein reveals the existence of epidemal growth factor-like domains linked to factor VIII-like sequences. Proc Natl Acad Sci USA 1990; 87: 8417-21.

10 Andersen MH, Graversen H, Fedosov SN, Petersen TE, Rasmussen JT. Functional analyses of two cellular binding domains of bovine lactadherin. Biochemistry 2000; 39: 6200-6.

11 Shi J, Heegaard CW, Rasmussen JT, Gilbert GE. Lactadherin binds selectively to membranes containing phosphatidyl-L-serine and increased curvature. Biochim Biophys Acta 2004; 1667: 82-90. 
12 Shi J, Gilbert GE. Lactadherin inhibits enzyme complexes of blood coagulation by competing for phospholipid binding sites. Blood 2003; 101: $2628-36$.

13 Shi J, Shi Y, Waehrens LN, Rasmussen JT, Heegaard CW, Gilbert GE. Lactadherin detects early phosphatidylserine exposure on immortalized leukemia cells undergoing programmed cell death. Cytometry A 2006; 69: 1193-201.

14 Yeung T, Gilbert GE, Shi J, Silvius J, Kapus A, Grinstein S. Membrane phosphatidylserine regulates surface charge and protein localization. Science 2008; 319: 210-13.

15 Gilbert GE, Drinkwater D, Barter S, Clouse SB. Specificity of phosphatidylserine-containing membrane binding sites for factor VIII: studies with model membranes supported by glass microspheres (Lipospheres). J Biol Chem 1992; 267: 15861-8.

16 Moore EW. Ionized calcium in normal serum, ultrafiltrates, and whole blood determined by ion-exchange electrodes. J Clin Invest 1970; 49: 318-34.

17 Gemmell CH, Sefton MV, Yeo EL. Platelet-derived microparticle formation involves glycoprotein-IIb-IIIa - inhibition by RGDS and a Glanzmann's thrombasthenia defect. $J$ Biol Chem 1993; 268: 14586-9.

18 Phillips JE, Gilbert GE. Platelet exposure of functional factor VIII binding sites requires stimulation by thrombin and shear stress. Blood 1995; 86: 549a.

19 Shi J, Gilbert GE, Kokubo Y, Ohashi T. Role of the liver in regulating numbers of circulating neutrophils. Blood 2001; 98: 1226-30.

20 Eitzman DT, Westrick RJ, Nabel EG, Ginsburg D. Plasminogen activator inhibitor-1 and vitronectin promote vascular thrombosis in mice. Blood 2000; 95: 577-80.

21 Gilbert GE, Drinkwater D. Specific membrane binding of factor VIII is mediated by O-phospho-L-serine, a moiety of phosphatidylserine. Biochemistry 1993; 32: 9577-85.

22 Comfurius P, Smeets EF, Willems GM, Bevers EM, Zwaal RFA. Assembly of the prothrombinase complex on lipid vesicles depends on the stereochemical configuration of the polar headgroup of phosphatidylserine. Biochemistry 1994; 33: 10319-24.

23 Gilbert GE, Arena AA. Activation of the factor VIIIa-factor IXa enzyme complex of blood coagulation by membranes containing phosphatidyl-L-serine. $J$ Biol Chem 1996; 271: 11120-5.

24 Gilbert GE, Arena AA. Partial activation of the factor VIIIa-factor IXa enzyme complex by dihexanoic phosphatidylserine at submicellar concentrations. Biochemistry 1997; 36: 10768-76.

25 Shao C, Novakovic VA, Head JF, Seaton BA, Gilbert GE. Crystal structure of lactadherin $\mathrm{C} 2$ domain at 1.7 angstrom resolution with mutational and computational analyses of its membrane-binding motif. J Biol Chem 2007; 283: 7230-41.

26 Munnix IC, Strehl A, Kuijpers MJ, Auger JM, van der Meijden PE, van Zandvoort MA, oude Egbrink MG, Nieswandt B, Heemskerk JW. The glycoprotein VI-phospholipase Cgamma2 signaling pathway controls thrombus formation induced by collagen and tissue factor in vitro and in vivo. Arterioscler Thromb Vasc Biol 2005; 25: 2673-8.

27 Pauer HU, Baird TR, Johnston K, Seaton BA, Furie B, Furie BC. Prothrombin fragment 1 and annexin $\mathrm{V}$ localize differently after laser induced vascular injury in vivo. J Thromb Haemost 2005; 3: Abstract OR374.
28 Andree H, Reutelingsperger C, Hauptmann R, Hemker H, Hermens W, Willems G. Binding of vascular anticoagulant $\alpha$ (VAC $\alpha$ ) to planar phospholipid bilayers. J Biol Chem 1990; 265: 4923-8.

29 Dachary-Prigent J, Freyssinet JM, Pasquet JM, Carron JC, Nurden AT. Annexin V as a probe of aminophospholipid exposure and platelet membrane vesiculation: a flow cytometry study showing a role for free sulfhydryl groups. Blood 1993; 81: 2554-65.

30 Reutelingsperger CPM, Hornstra G, Hemker HC. Isolation and partial purification of a novel anticoagulant from arteries of human umbilical cord. Eur J Biochem 1985; 151: 625-9.

31 Tait JF, Sakata MS, McMullen BA, Miao CH, Funakoshi T, Hendrickson LE, Fujikawa K. Placental anticoagulant proteins: isolation and comparative characterization of four members of the lipocortin family. Biochemistry 1988; 27: 6268-76.

32 London F, Ahmad SS, Walsh PN. Annexin V inhibition of factor IXa-catalyzed factor $\mathrm{X}$ activation on human platelets and on negatively-charged phospholipid vesicles. Biochemistry 1996; 35: 16886-97.

33 Dasgupta SK, Guchhait P, Thiagarajan P. Lactadherin binding and phosphatidylserine expression on cell surface - comparison with annexin A5. Transl Res 2006; 148: 19-25.

34 Romisch J, Seiffge D, Reiner G, Paques EP, Heimburger N. In-vivo antithrombotic potency of placenta protein 4 (annexin V). Thromb Res 1991; 61: 93-104.

35 Van Ryn-McKenna J, Merk H, Muller TH, Buchanan MR, Eisert WG. The effects of heparin and annexin $\mathrm{V}$ on fibrin accretion after injury in the jugular veins of rabbits. Thromb Haemost 1993; 69: 227-30.

36 Thiagarajan P, Benedict CR. Inhibition of arterial thrombosis by recombinant annexin V in a rabbit carotid artery injury model. Circulation 1997; 96: 2339-47.

37 Tracy P, Peterson J, Nesheim M, McDuffie F, Mann K. Interaction of coagulation factor V and factor Va with platelets. J Biol Chem 1979; 254: 10354-61.

38 Swords NA, Tracy PB, Mann KG. Intact platelet membranes, not platelet-released microvesicles, support the procoagulant activity of adherent platelets. Arterioscler Thromb 1993; 13: 1613-22.

39 George J, Thoi L, McManus L, Reimann T. Isolation of human platelet membrane microparticles from plasma and serum. Blood 1982; 60: $834-40$

40 Gilbert GE, Sims PJ, Wiedmer T, Furie B, Furie BC, Shattil SJ. Platelet-derived microparticles express high affinity receptors for factor VIII. J Biol Chem 1991; 266: 17261-8.

41 Bevers E, Wiedmer T, Comfurius P, Shattil S, Weiss H, Zwaal R, Sims $\mathrm{P}$. Defective $\mathrm{Ca} 2+$-induced microvesiculation and deficient expression of procoagulant activity in erythrocytes from a patient with a bleeding disorder: a study of the red blood cells of Scott syndrome. Blood 1992; 79: $380-8$.

42 Kempton CL, Hoffman M, Roberts HR, Monroe DM. Platelet heterogeneity: variation in coagulation complexes on platelet subpopulations. Arterioscler Thromb Vasc Biol 2005; 25: 861-6.

43 Jobe SM, Wilson KM, Leo L, Raimondi A, Molkentin JD, Lentz SR, Di Paola J. Critical role for the mitochondrial permeability transition pore and cyclophilin D in platelet activation and thrombosis. Blood 2008; 111: 1257-65. 